\title{
Laudatio for Keti Tenenblat
}

\author{
Manfredo P. do Carmo
}

Keti Tenenblat was my first student at IMPA. At that time, 1970, she was a very energetic woman (those that are acquainted with her know this changed very little with time). During her thesis, she was teaching at the Federal University of Rio de Janeiro (which is quite far from IMPA), and was carrying her first child. In addition, I also asked her to use differential forms to give a proof that the structure equations of Elie Cartan were sufficient to immerse locally an $n$-dimensional Riemannian manifold into the $(n+k)$-Euclidean space. This turned out to be a interesting and stimulating paper that was recently used by B. Daniel in his work on $n$-dimensional homogeneous manifolds.

Keti Tenenblat completed her doctoral work in 1972. In 1973, she accepted a position at the University of Brasilia, where she stayed until now. The period from 1975 to 1978 was spent at the University of California, Berkeley, with a post-doctoral fellowship under the guidance of S.S. Chern.

One of the characteristics of Chern was his familiarity with classical geometry and his ability to find new and interesting problems in somewhat neglected areas. At the time, he was interested in Bäcklund transformations of surfaces. With her usual energy and enthusiasm, Keti embarked on this new challenge, and this turned out to be her favorite topic of research in the following years. Together with C.L. Terng she wrote a pioneering work on the subject that was published in the Annals of Mathematics in 1980.

Keti also worked on a variety of other subjects. For lack of space, I will not go into this. Due to the excellence of her scientific work, she was elected to the Brazilian Academy of Sciences in 1991 and to the Academy of Sciences for the Developing World (TWAS) in 2005.

Parallel to her research work, she furthered the development of Mathematics in Brazil. I will only mention a few examples: Head of the Mathematics Department of the University of Brasilia, for two years, Member of the Mathematics Committee of the National Research Council and Chairperson of the Mathematics Section of the Coordination of Postgraduate Studies of 
the Ministry of Education for several years, President of the Brazilian Mathematical Society for two years; these are only a few of the versatile activities through which she had a strong influence on the growth of Mathematics in Brazil.

I am proud to have been the advisor of Keti Tenenblat, mathematician and friend, and I am glad to join her worldwide friends in this volume which is the best tribute a mathematician can receive.

Manfredo P. do Carmo

IMPA

Rio de Janeiro

Brazil

e-mail: manfredo@impa.br 